\title{
A Memetic Algorithm for Single-orbit Image Scheduling of Agile Satellite
}

\author{
Dengyi Zhang ${ }^{1, a}$, Lei Guo ${ }^{1, b}$ \\ ${ }^{1}$ School of computer, Wuhan University, Wuhan 430072, China; \\ adyzhangwhu@163.com, bguolei@whu.edu.cn
}

Keywords: agile satellite, scheduling, memetic algorithm, local search

\begin{abstract}
This article analyzes the complex time constraints such as time window visibility and attitude transition time of the single-orbit image scheduling problem for agile satellite, establishes a constrained optimization model and proposes a memetic algorithm to tackle this NP-hard problem. In our algorithm, binary and permutation representation is combined to represent selecting and timetabling sub problem respectively, mixture of feasible and infeasible solutions are allowed to maintain population diversity, tabu search is introduced after crossover and mutation to improve local optimum. In the aspect of constraint handling, a repair method along with penalty function is developed. Experimental results show that this algorithm is a feasible approach for solving the above problem.
\end{abstract}

\section{Introduction}

The new generation of agile satellite such as Pleiades- 1 has three degree of freedom (roll, pitch, and yaw) for acquiring images, which provides possibility of getting more spot targets in single orbit. The single-orbit image scheduling problem (SOISP) of an agile satellite consists of selecting and timetabling a subset of images among a given set of candidates to maximize a gain function while satisfy imperative constraints.

The problem has a significant economic importance and is proved to be NP-hard, making it an attractive research area. Lemaitre and Verfaillie [1] (2002) first proposed this problem, they set out the overall selecting and scheduling problem of French Pleiades-1 satellite and analyzed its difficulties, and then presented four different methods: greedy algorithm, dynamic programming algorithm, constraint programming and local search. Habet and Vasquez [2] (2004) proposed a $\mathrm{CN}-\mathrm{Tabu}$ search algorithm, the CN-Tabu algorithm adopted consistent and saturated neighborhood to prevent infeasible solution and search optimal solution, and hybrided a systemetic search using partial enumerations. Cordeau and Laporte [3] (2005) proposed a unified tabu search algorithm, except for constraint relaxation to allow violations of time window constraint, the algorithm combined four diversification mechanisams: contionous, perturbation, intra-sequence and restrats. In [4] (2010), a dynamic programming algorithm is proposed on a relaxed problem to calculate upper bounds. Tangpattanakul and Jozefowiez [5,6] $(2013,2015)$ presented a biased random key genetic algorithm (BRKGA) and an indicator-based multi-objective local search (IBMOLS).

In this paper, to solve the SOISP above, a constrained optimization model is built and a memetic algorithm is proposed. In our algorithm, using two decision variable to represent selecting and timetabling sub problem respectively, allowing infeasible solutions to maintain population diversity, after crossover and mutation, a local search procedure is introduced to improve local search capability.

\section{Problem description and formulation}

The single-orbit image scheduling problem of an agile satellite can be simply described as follows: given a set of candidate images $I$ which could be imaged in a single orbit ,each image $i \in I$ has a weight $W_{i}$ refelcting its improtance, it can be captured between earliest start time and latest start time (we call it time window visibility constraint, see Fig.1(a)), and attitude transition time is needed between consecutive images (we call it transition constraint, see Fig.1(b)). For stereoscopic request, 
the corresponding image need to be acqured twice in different azimuth angle (we call it stereoscopic constraint). The objective is to select a subset of images among the candidates and timetabling their start times to maximize the sum weights while satisfy above constraints.

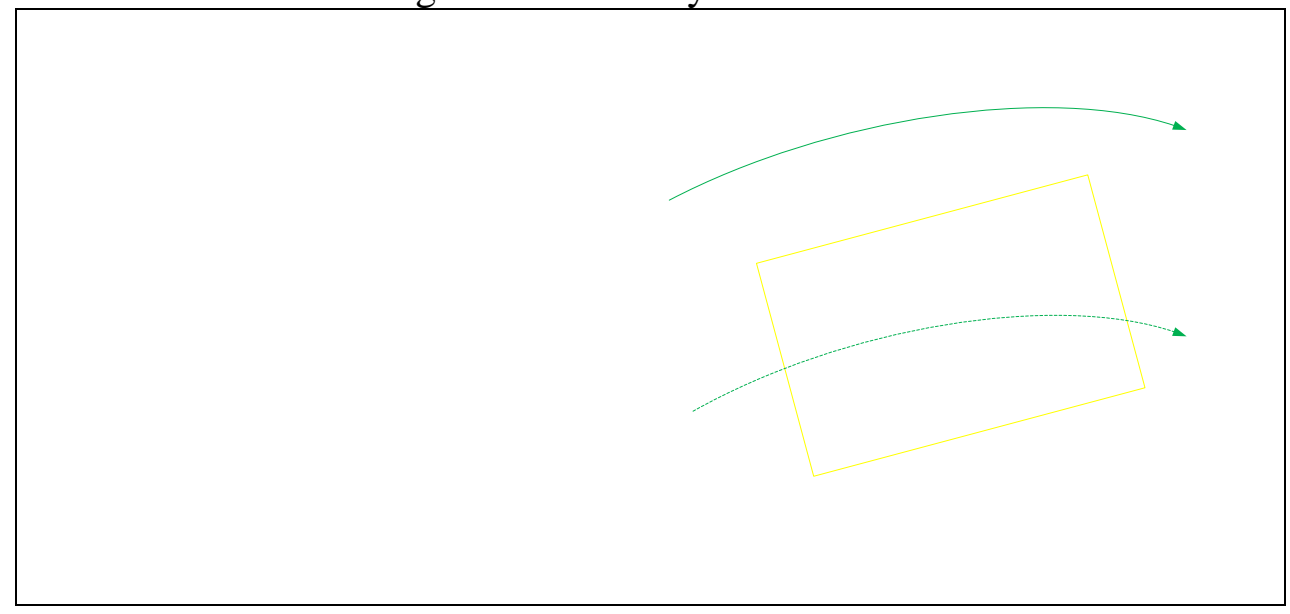

Fig.1 Time window visibility and transition time of multiple spot targets to be imaging The problem can be described formally as follows [1]:

\section{1) Basic data}

For each candidate image $i \in I, n=|I|$, let

$E_{i}$ be its earliest start time;

$L_{i}$ be its latest start time;

$D_{i}$ be its duration;

$T w_{i}$ be its twin images according to stereoscopic request;

$W_{i}$ be its weight.

For each possible pair of images $(i, j), i \neq j, \operatorname{Tr}_{i j}$ denotes the minimum transition time from the end of $i$ to the beginning of $j$. If there is not enough time to transition between $i$ and $j$, a default very large value will be given.

2) Decision variables

Two variables are defined for each image $i$ :

$x_{i} \in\{0,1\}$ equals 1 if the image $i$ is selected, and 0 otherwise;

$t_{i}$ is the shooting start time of the acquisition of the image $i$, if selected. The value of this variable is irrelevant when $x_{i}=0$.

The $t_{i}$ values allow to give the order of the selected images and to schedule the images in time.

One binary variable is associated to each possible pair of images $(i, j), i \neq j$ :

$y_{i j} \in\{0,1\}$ equals 1 if $i$ is followed by $j$ in the selected sequence, 0 otherwise.

3) Constraints

$$
\begin{aligned}
& \forall i \in I,\left(x_{i}=1\right) \Rightarrow\left(E_{i} \leq t_{i} \leq L_{i}\right) \\
& \forall i, j \in I,\left(y_{i j}=1\right) \Rightarrow\left(t_{i}+D_{i}+T r a_{i j} \leq t_{j}\right) \\
& \forall i \in I,\left(T w_{i} \neq 0\right) \Rightarrow\left(x_{i}=x_{T w_{i}}\right) \\
& \sum_{i \in I} y_{0 i} \leq 1 \text { and } \sum_{i \in I} y_{i n} \leq 1
\end{aligned}
$$

Equation (1) states the time window for the start time of a candidate image. Start times of consecutive images must respect minimum transition times (2). Stereoscopic constraints are satisfied by (3). There is only one start image and one last image (4).

4) Objective function

$$
\max f(x)=\sum_{i \in I} W_{i} \times x_{i}
$$

The objective function is to maximize the total gain of selected images. 


\section{A memetic algorithm for SOISP}

Memetic algorithm [7] is a swarm intelligence optimization algorithm based on human cultural evolution strategy, introducing local search into genetic algorithm, so-called "hybrid genetic algorithm." Its search process is similar to the genetic algorithm, first initialize the population, followed by iterative implementation of selection, crossover and mutation operators, the difference is that for memetic algorithm local search are carried out after certain iterations, so not only it retains the global optimization capacity of genetic algorithm, and improves the quality of the solution by local search, reduces the number of iterations so as to accelerate the speed of evolution. In memetic algorithms, local search strategy is critical, it directly affects the operational efficiency of the algorithm. Next we will describe the key part of our proposed memetic algorithm.

\section{1) Representation}

Because the single-orbit image scheduling problem of an agile satellite includes selection and ordering two sub-problems, so a hybrid binary and permutation representation are adopted. The binary vector $X$ represents the selection sub-problem, and its length is the number of images equals to $n, x_{i}$ denotes the ith image, the value of 1 means it was selected, and 0 otherwise. $Y$ is a permutation of $n$ coding for ordering sub-problems. For example, suppose there are five candidate images numbered $1,2,3,4,5$, then the representation $\{X=(0,1,0,1,1), Y=(4,3,1,5,2)\}$ denotes that images $2,4,5$ are selected for acquiring, the imaging order is 4,5,2 . This kind of representation leads the algorithm to search the whole unconstrained search space, which allows infeasible even illegal solution. Though the solution is not feasible, it increases the diversity of the population and may guide the search for getting the optimal solution.

\section{2) Initialization}

Numerous studies show that, in order to ensure the effective implementation of the algorithm, the initial solution must be feasible, legal, have good benefits and diversity. Therefore a greedy random insertion algorithm is used to generate initial population. The basic idea is as follows: images are sorted by their weight and earliest start time. The greedy algorithm selects images with higher weight one by one according to their start time, when there are images with the same weight, randomly selects one inserting into the image sequence. Remember that all selected images must satisfy the above constraints.

\section{3) Fitness computation}

This algorithm allows infeasible solution, so fitness function is defined as

$$
F(x)=f(x)-a w(x)
$$

In (6), $f(x)$ is the total gain of selected images, $w(x)$ is the total number of time window violation, parameter $\alpha$ is initially set to 1 , and adaptive changes during the search. More precisely, each iteration process, if the current solution is not feasible $\alpha=\alpha^{*}(1+\beta)$, otherwise $\alpha=\alpha /(1+\beta), \beta$ is a random number between 0 and 1 .

4) EA operators

Selection: choose parent using binary tournament selection method. Every two randomly selected individuals are compared for their fitness values, choose the one with a larger fitness value as a parent, the process is repeated until the parents fill the mating pool.

Crossover: crossover operator has many implementation patterns such as One-point crossover, Two-point crossover, and Uniform crossover. In this paper, Two-point uniform crossover is used for the binary vector $X$. For the permutation $Y$, in order better to retain a partial chromosome arrangement and can integrate other ordered structure, we use partially mapped crossover method (PMX).

Mutation: for the vector $X$, using the basic mutation operator which the value of variation point was reversed. For the permutation $Y$, randomized block mutation operator is adopted, each block contains from 2 to 5 genes, exchanging two blocks are carried out with a certain probability.

5) Constraints handling

Application of the above designed operator will generate infeasible solution that violates the constraints (1) (2) (3). In this paper, a strategy of mixed repair method and penalty function is combined. The penalty function has been introduced above; the repair method is described below. 
After each 100 iterations, a reordering of the images in the solution is performed in order to meet the constraints (1) (2) (3) and to maximize the objective function (5), each image is removed and reinserted into its best position, if image cannot satisfy the above constraint, it will be deleted.

6) Local search

The local search used in this algorithm is based on the classical add and remove operator. Its neighborhood structure can be described in Fig.2. After 1 or 2 images (randomly chosen) are removed in the current schedule, 1 or 2 unselected images are added which can maximize the fitness value.

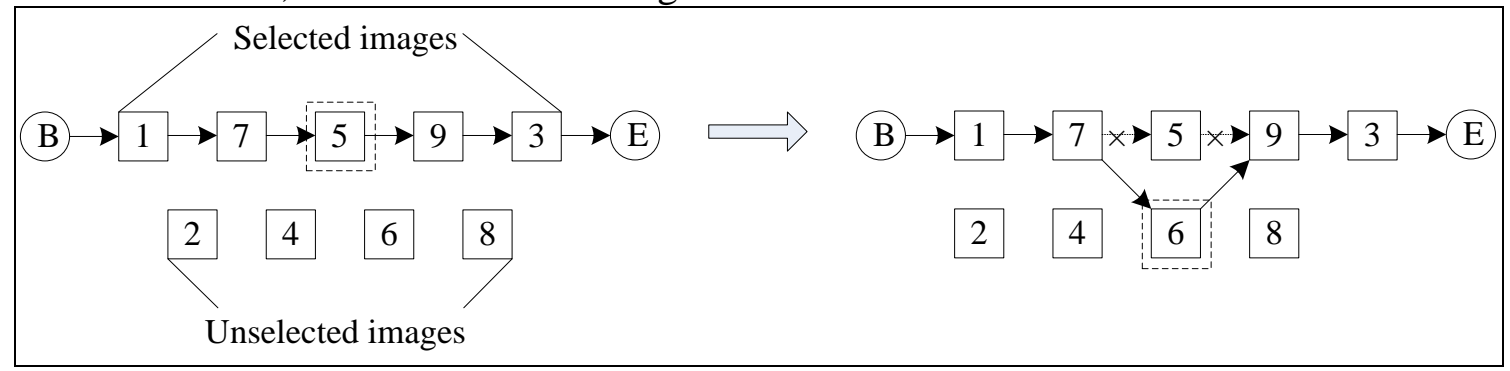

Fig.2 Neighborhood structure of local search

Tabu list is introduced to avoid circulation. When an image is added into or removed from the schedule, it is forbidden to move (removal or reinsertion) for certain iterations. Aspiration criterion is that if the move can get a larger profit than the best solution found so far, it is not forbidden.

\section{Computational results}

The selected satellite is the latest high-resolution agile satellite Pleiades-1A, its main parameters are shown in Tab.1.The candidate images are uniformly distributed along the ground track of the satellite with a quantity various from 100 to 400, their weights are randomly chosen in $[1,10]$. Computational results are show in Tab.2 with comparisons with the local search (LA) algorithm in [1] and the biased random key genetic algorithm (BRKGA) in [5].

Table 1 Main parameters of Pleiades-1A

\begin{tabular}{cc}
\hline Satellite name & Pleiades-1A \\
\hline Launch time (year) & 2011 \\
Orbit height $/ \mathrm{km}$ & 695 \\
Imaging width $/ \mathrm{km}$ & 20 \\
Maneuver ability $/\left(^{\circ}\right)$ & \pm 60 \\
Transition speed & $60\left(^{\circ}\right) / 25 \mathrm{~s}$ \\
\hline
\end{tabular}

Table 2 Comparisons of diferrent algorithms in gain and number of selected images

\begin{tabular}{|c|c|c|c|c|c|c|}
\hline \multirow[t]{2}{*}{ Number of images } & \multicolumn{3}{|c|}{ Gain of selected images } & \multicolumn{3}{|c|}{ Number of selected images } \\
\hline & Our algorithm & BRKGA & LA & Our algorithm & BRKGA & LA \\
\hline 100 & 398 & 396 & 392 & 54 & 53 & 52 \\
\hline 200 & 534 & 530 & 526 & 83 & 81 & 80 \\
\hline 300 & 744 & 736 & 713 & 124 & 120 & 115 \\
\hline 400 & 867 & 825 & 804 & 156 & 151 & 144 \\
\hline
\end{tabular}

From Tab.2, we can see that the results of our memetic algorithm is best compared with BRKGA and LA both in gain and number of selected images, which shows that our algorithm is a competitive algorithm to solve the single-orbit image scheduling problem of agile satellite.

\section{Conclusion}

In this paper, a constrained optimization model which involves time window visibility and attitude transition constraint was firstly presented to formulate the single-orbit image scheduling problem of agile satelllite. We then proposed a memetic algorithm which introduced a tabu local search after EA operators to tackle this NP-hard problem. In our algorithm, a mixture representation of binary and permutation was used, infeasible solutions were allowed of violating only the time window constraint in hope to improve the optimum. In the aspect of constraint handling, a repair method along with 
penalty function was developed. Test results showed that this algorithm is a feasible approach for solving the problem.

\section{References}

[1]Lemaître M, Verfaillie G, Jouhaud F, et al. Selecting and scheduling observations of agile satellites[J]. Aerospace Science and Technology, 2002, 6(5): 367-381.

[2]Habet D, Vasquez M. Solving the selecting and scheduling satellite photographs problem with a consistent neighborhood heuristic[C].2004 16th IEEE International Conference on Tools with Artificial Intelligence. IEEE, 2004: 302-309.

[3]Cordeau J F, Laporte G. Maximizing the value of an earth observation satellite orbit[J]. Journal of the Operational Research Society, 2005, 56(8): 962-968.

[4]Habet D, Vasquez M, Vimont Y. Bounding the optimum for the problem of scheduling the photographs of an Agile Earth Observing Satellite[J]. Computational Optimization and Applications, 2010, 47(2): 307-333.

[5]Tangpattanakul P, Jozefowiez N, Lopez P. Biased random key genetic algorithm with hybrid decoding for multi-objective optimization[C] 2013 Federated Conference on Computer Science and Information Systems. IEEE, 2013: 393-400.

[6]Tangpattanakul P, Jozefowiez N, Lopez P. A multi-objective local search heuristic for scheduling Earth observations taken by an agile satellite[J]. European Journal of Operational Research, 2015, 245(2): 542-554.

[7]Krasnogor N. Memetic algorithms[M]. Springer Berlin Heidelberg, 2012: 905-935. 